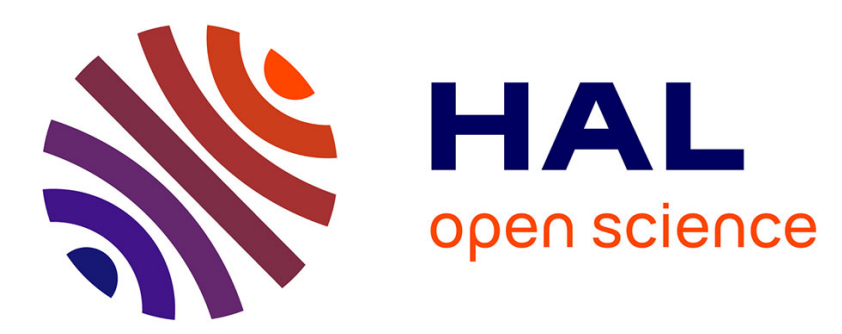

\title{
All-fiber spectral compression of picosecond pulses at telecommunication wavelength enhanced by amplitude shaping
}

Julien Fatome, Bertrand Kibler, Esben Ravn Andresen, Herve Rigneault, Christophe Finot

\section{To cite this version:}

Julien Fatome, Bertrand Kibler, Esben Ravn Andresen, Herve Rigneault, Christophe Finot. All-fiber spectral compression of picosecond pulses at telecommunication wavelength enhanced by amplitude shaping. Applied optics, 2012, 51 (19), pp.4547-4553. 10.1364/AO.51.004547 . hal-00699103

\section{HAL Id: hal-00699103 https://hal.science/hal-00699103}

Submitted on 19 May 2012

HAL is a multi-disciplinary open access archive for the deposit and dissemination of scientific research documents, whether they are published or not. The documents may come from teaching and research institutions in France or abroad, or from public or private research centers.
L'archive ouverte pluridisciplinaire HAL, est destinée au dépôt et à la diffusion de documents scientifiques de niveau recherche, publiés ou non, émanant des établissements d'enseignement et de recherche français ou étrangers, des laboratoires publics ou privés. 


\title{
All-fiber spectral compression
}

\author{
of picosecond pulses
}

\section{at telecommunication wavelength}

\section{enhanced by amplitude shaping}

\author{
Julien Fatome ${ }^{1}$, Bertrand Kibler ${ }^{1}$, Esben R. Andresen ${ }^{2}$, \\ Hervé Rigneault ${ }^{2}$ and Christophe Finot ${ }^{1 *}$ \\ ${ }^{1}$ Laboratoire interdisciplinaire Carnot de Bourgogne, UMR 5209 CNRS - Université de \\ Bourgogne, Dijon, France \\ ${ }^{2}$ Institut Fresnel, UMR 6133 CNRS, Aix-Marseille Université, Domaine Universitaire de St \\ Jérôme 13397 Marseille Cedex 20, France \\ *Corresponding author: christophe.finot@u-bourgogne.fr
}

We demonstrate efficient spectral compression of picosecond pulses in an all-fiber configuration at telecommunication wavelengths. A spectral compression by a factor 12 is achieved. Performing temporal shaping with a parabolic pulse significantly improves the spectral compression with much lower substructures and an enhanced Strehl ratio.

OCIS codes: 190.4370 (nonlinear optics, fibers), 320.5540 (pulse shaping), 070.4340 (optical signal processing) 


\section{Introduction}

Optical fibers are an ideal medium to observe the consequences of Kerr nonlinearity. Indeed, thanks to the guided propagation and light confinement, the low third-order nonlinear coefficient of silica is counterbalanced by a large available interaction distance. Therefore, optical fibers have been widely used to observe large self-phase modulation (SPM) of transform-limited pulses, which usually leads to a spectral expansion of the pulse [1]. If this effect has ordinarily a negative impact on long-haul telecommunications or high-power fiber amplifiers, various specific applications may on the contrary benefit from this spectral enhancement. For example, highly-coherent multi-wavelength continuum sources have been proposed spanning the whole band of conventional optical telecommunications [2]. Another widely used application is nonlinear temporal pulse compression and relies on the linear compensation of the nonlinear chirp induced by SPM. Thanks to this technique, femtosecond few-cycles pulses have been generated [3]. However, the quality of the results depends directly on the temporal intensity profile of the initial pulses. Therefore, it has been shown that initial pulse shaping may significantly improve the performance of these devices [4-6] by taking advantage of the parabolic pulse features [7].

A less-known potential application of SPM (even if it was described as soon as 1978 [1]) relies on the configuration where the initial pulse is negatively linearly chirped corresponding to an anomalous dispersive medium. In such a configuration, the SPM-induced chirp cancels the initial frequency modulation and long and short wavelengths redistribute toward the center wavelength, leading to a spectral narrowing of the pulse [8-11]. Concentrating the energy in the central part of the spectrum has for example been found of practical interest for the generation of high power picosecond pulses from femtosecond oscillators [12] or for biophotonics applications 
with an enhanced spectral resolution [13]. However, once again, the performances of the spectral compression process crucially depends on the initial pulse shape and routinely used hyperbolic secant or Gaussian temporal intensity profiles lead to the presence of large spectral pedestals. To overcome this issue, the optimum pulse shape has been found to be theoretically parabolic [14, 15], which has recently been experimentally validated by using femtosecond pulses evolving in a passive photonic crystal fiber around $1 \mu \mathrm{m}[16]$.

In this contribution, we provide a new illustration of the benefits brought by an initial shaping of the temporal intensity profile. Our experiments explore a completely different range of input parameters (initial wavelength, duration and peak power as well as typical fiber lengths) and a high-quality spectral compression by a factor above 10 is demonstrated in a passive fiber. We first recall the basis of spectral narrowing and quantify the process by means of numerical simulations. We then describe our all-fiber experimental setup and discuss the experimental results that have been obtained.

\section{Principle of operation and numerical simulations}

Spectral narrowing occurs when a linearly chirped pulse (with a chirp provided by an anomalous dispersion medium such as a pair of gratings $[8,12]$, a prism $[10,11]$ or an hollow-core photonic bandgap fiber [17]) is affected by self-phase modulation in a highly nonlinear Kerr medium. Basically, if the initial pulse has the temporal complex field envelope $\psi$ given by:

$$
\psi(T, 0)=\sqrt{P(T, 0)} \exp \left(-i \frac{C_{T}}{2} T^{2}\right)
$$


with $\mathrm{P}$ being the temporal intensity profile and $\mathrm{C}_{\mathrm{T}}$ the chirp parameter, then the SPM induced after a propagation length $\mathrm{L}$ is given by the following nonlinear term that adds to the initial phase, $\gamma$ is respectively the nonlinear coefficient of the fiber under test :

$$
\varphi_{N L}(T, L)=\gamma P(T, 0) L
$$

This results in the following pulse (here the impact of other effects such as dispersion or attenuation has been neglected) :

$$
\psi(T, L)=\sqrt{P(T, 0)} \exp \left(-i\left(\frac{C_{T}}{2} T^{2}-\gamma P(T, 0) L\right)\right)
$$

From this simple equation, it is clear that starting from a linearly chirped pulse, one can obtain a transform-limited pulse of the same duration (therefore with a narrower spectrum) if this condition is fulfilled:

$$
\frac{C_{T}}{2} T^{2}-\gamma P(T, 0) \quad L=K
$$

$\mathrm{K}$ being a constant. If this requirement is not fulfilled, the input chirp compensation will only be partial with a time-dependent term, resulting in the large spectral pedestals that are usually observed [10, 12, 17].

Following Eq. (4), it is important to note that this last condition is perfectly fulfilled for an input temporal intensity profile having a parabolic shape :

$$
P(T, 0)=\frac{C_{T}}{2 \gamma L} T^{2}-K
$$


that can be written using the usual notation for a parabolic bright pulse as (for $|\mathrm{T}|<\mathrm{T}_{\mathrm{P}}$ ) :

$$
P(T, 0)=P_{P}\left(1-\frac{T^{2}}{T_{P}^{2}}\right)
$$

with $P_{P}=-K$ being the peak power of the pulse and $T_{P}$ being the half-total width of the parabolic pulse such that

$$
T_{P}^{2}=-\frac{2 \gamma L P_{P}}{C_{T}}
$$

We can make out that $\mathrm{K}$ should be negative and that a negative input chirp coefficient $C_{T}$ is needed. From (7) it also appears that the peak power of the pulses is directly proportional to the chirp coefficient and inversely proportional to the length of the fiber and to the nonlinear coefficient. Note that using dips of light on a continuous background could also be a possible solution and dark parabolic profiles [18] can also fulfill Eq. (5) to spectrally compress positively chirped pulse.

In order to get more precise insights into the nonlinear dynamics of the spectral compression, we numerically integrate the standard nonlinear Schrödinger equation that takes into account also the second order dispersion of the fiber $\beta_{2}$ :

$$
i \frac{\partial \psi}{\partial z}=\frac{\beta_{2}}{2} \frac{\partial^{2} \psi}{\partial T^{2}}-\gamma|\psi|^{2} \psi
$$

In the following, we base our discussion on the experimental parameters that will be further detailed in section 3. We will compare hyperbolic secant and parabolic pulses having a 
full width at half-maximum (FWHM) temporal duration of $70 \mathrm{ps}$ and a linear chirp coefficient $\mathrm{C}_{\mathrm{T}}$ of $-1.93 \mathrm{GHz} / \mathrm{ps}$. Both pulses have a similar FWHM spectral width of $130 \mathrm{GHz}$. The spectral compression will take place in an optical fiber having the following properties: $\beta_{2}=9.610^{-4}$ $\mathrm{ps}^{2} / \mathrm{m}, \gamma=10 / \mathrm{W} / \mathrm{km}$ and a fixed length $\mathrm{L}$ of $1 \mathrm{~km}$.

Figure 1 describes the evolution of the output spectral profiles as a function of the input pulse peak-power for the two pulse shapes under investigation (hyperbolic secant and parabolic pulses). This figure outlines the significant influence of the initial shape on the compression behavior. If the spectral width and the power required for optimum compression seem comparable in both cases, the presence of sidelobes and oscillations is clearly accentuated in the case of a sech ${ }^{2}$ profile. One can also make out that the spectral brightness is enhanced using a parabolic input pulse. The spectral expansion following the point of optimum compression also differs significantly.
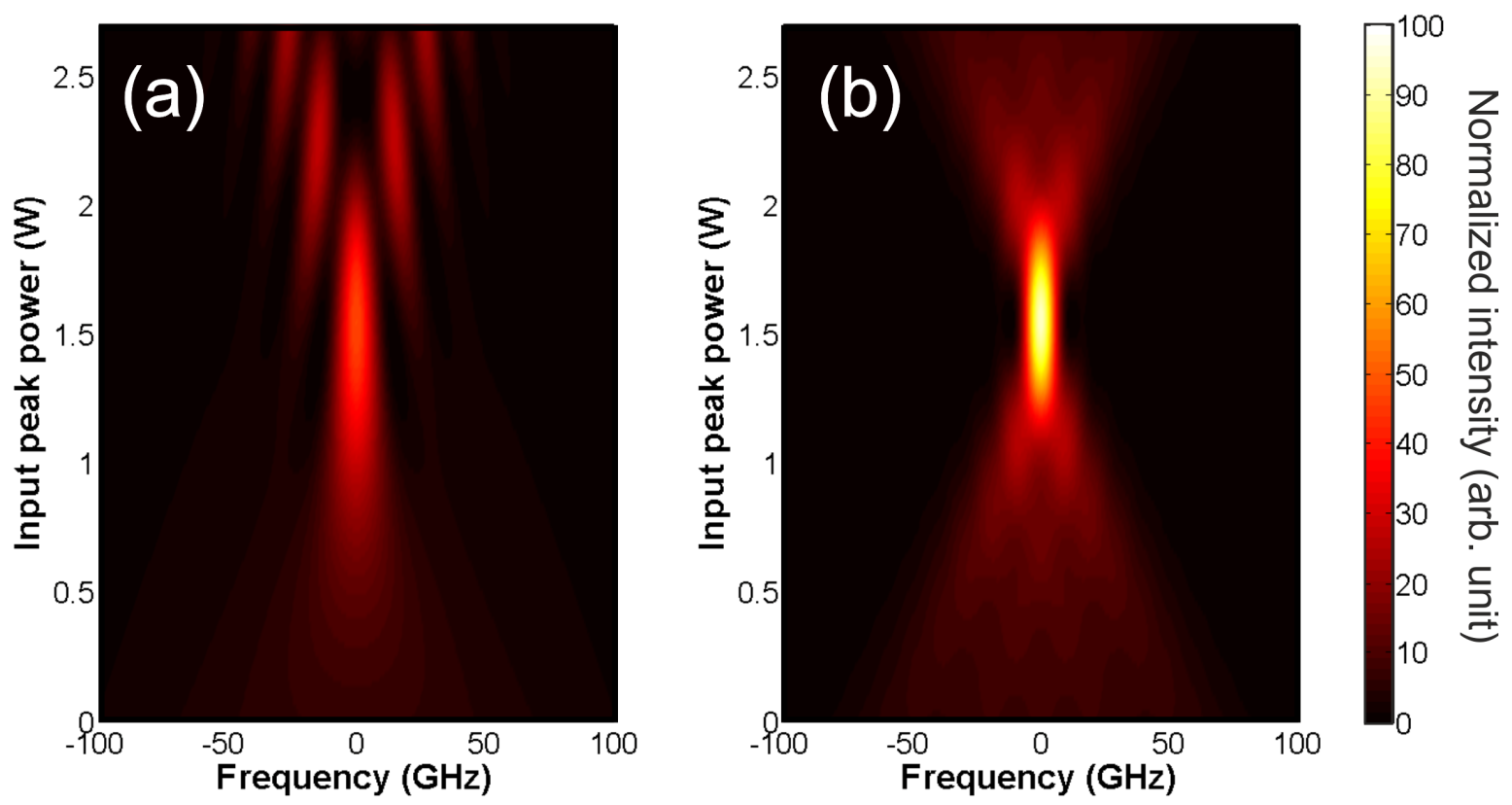

Fig. 1 : Simulation: Evolution of the output spectral profile according to the input peak power for an initial sech ${ }^{2}$ intensity profile (a) and for a parabolic intensity profile (b). Spectra are normalized so that the energy of the spectra equals 1 . 
Figure 2 describes more quantitatively the differences between the two initial pulse shapes. More precisely, we have plotted the evolution of two quantities as a function of the input peak-power: the FWHM of the optical spectrum (Fig. 2a) and the Strehl ratio (Fig. 2b) that we use in order to assess the quality of the spectral compression. This Strehl ratio, often used to assess the spatial performance of imaging system, can also be used to quantify the quality of a temporal compression [19]. In our case, we define the Strehl ratio as the ratio between the actual spectral peak power and the spectral peak power obtained assuming a flat temporal phase of the pulse. Therefore, the Strehl ratio is comprised between 0 and 1, 1 defining an ideal spectral compression. From the results of these two figures, we can make out several important conclusions. First, we can note that the optimum compression (both in terms of spectral width and Strehl ratio) is obtained for an input peak power of $1.55 \mathrm{~W}$ for sech and parabolic pulse shapes, respectively. This value corresponds to input pulse energies of 105 and $125 \mathrm{pJ}$ respectively and is in agreement with the prediction of Eq. (8). In both cases, a compression factor close to 13 is achieved and one can note that between $1.2 \mathrm{~W}$ and $1.8 \mathrm{~W}$ of input peakpower, the FWHM spectral width does not exhibit major differences. Therefore, the main parameter to definitively assess the best configuration and power will be the Strehl ratio. A significantly higher Strehl ratio is indeed obtained in the case of parabolic pulses, with value as high as 0.99 , synonym of transform-limited pulses. It has to be compared to the performances achieved with a picosecond sech pulse with a poor resulting Strehl ratio of 0.24.

Interestingly, in the case of a parabolic pulse, the root-mean-square (rms) width of the pulse spectrum according to the input peak power follows a simple parabolic law. This result fully agrees with the evolution of the temporal rms width in a dispersive medium : in [20], it has indeed been shown that the temporal rms of any pulse propagating in a dispersive medium (i.e. a 
medium that imposes a spectral parabolic phase) scales following a non-trivial parabolic law. In the case of a parabolic pulse, we can clearly draw a parallel between the impact of the temporal parabolic SPM-induced phase and the action of second order dispersion acting as a parabolic phase in the spectral domain [15].
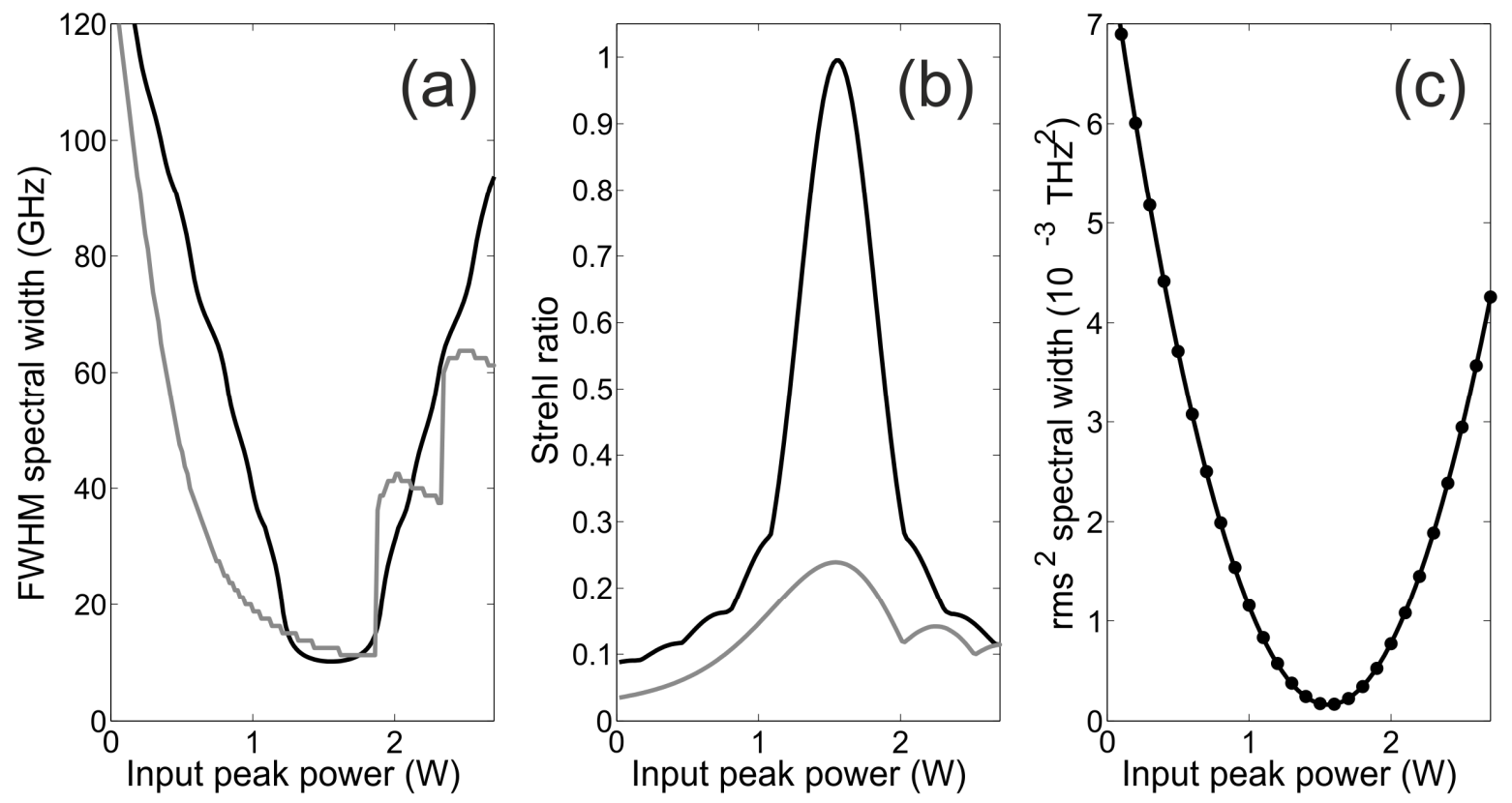

Fig. 2 : Simulation: Evolution of the output spectral parameters according to the initial peak-power for the parabolic pulse shape (solid black line) and sech pulse ( grey line)

(a) FWHM spectral width. (b) Strehl ratio. (c) Evolution of the rms spectral width as a function of input peak power for a parabolic pulse : numerical results (solid black line) are compared to a parabolic fit (dots).

Details of the output temporal and spectral intensity and chirp profiles generated at the optimum compression point are shown in Fig. 3. The temporal features of the spectrally compressed pulse confirm that for an initial parabolic shape, a null temporal chirp is obtained, synonym of the expected flat phase. On the contrary, a non-negligible residual chirp is visible for the hyperbolic secant pulse. Comparing the initial and compressed pulses, we can note that there is no change in the temporal intensity profile, thus confirming that the impact of chromatic dispersion is here negligible. Regarding the details of the compressed spectral profile (Fig. 3c), 
as already described in [15], the parabolic shape leads to significantly lower sidelobes, with a very low ratio of energy contained in the wings of the spectrum. The recompressed spectral profile can be theoretically described by a Bessel function of the first kind [15]. In the case of the hyperbolic pulse, some noticeable sidelobes are visible containing a non-negligible part of the pulse energy. Consequently, the spectral peak intensity is twofold under the one achieved with a parabolic pulse.
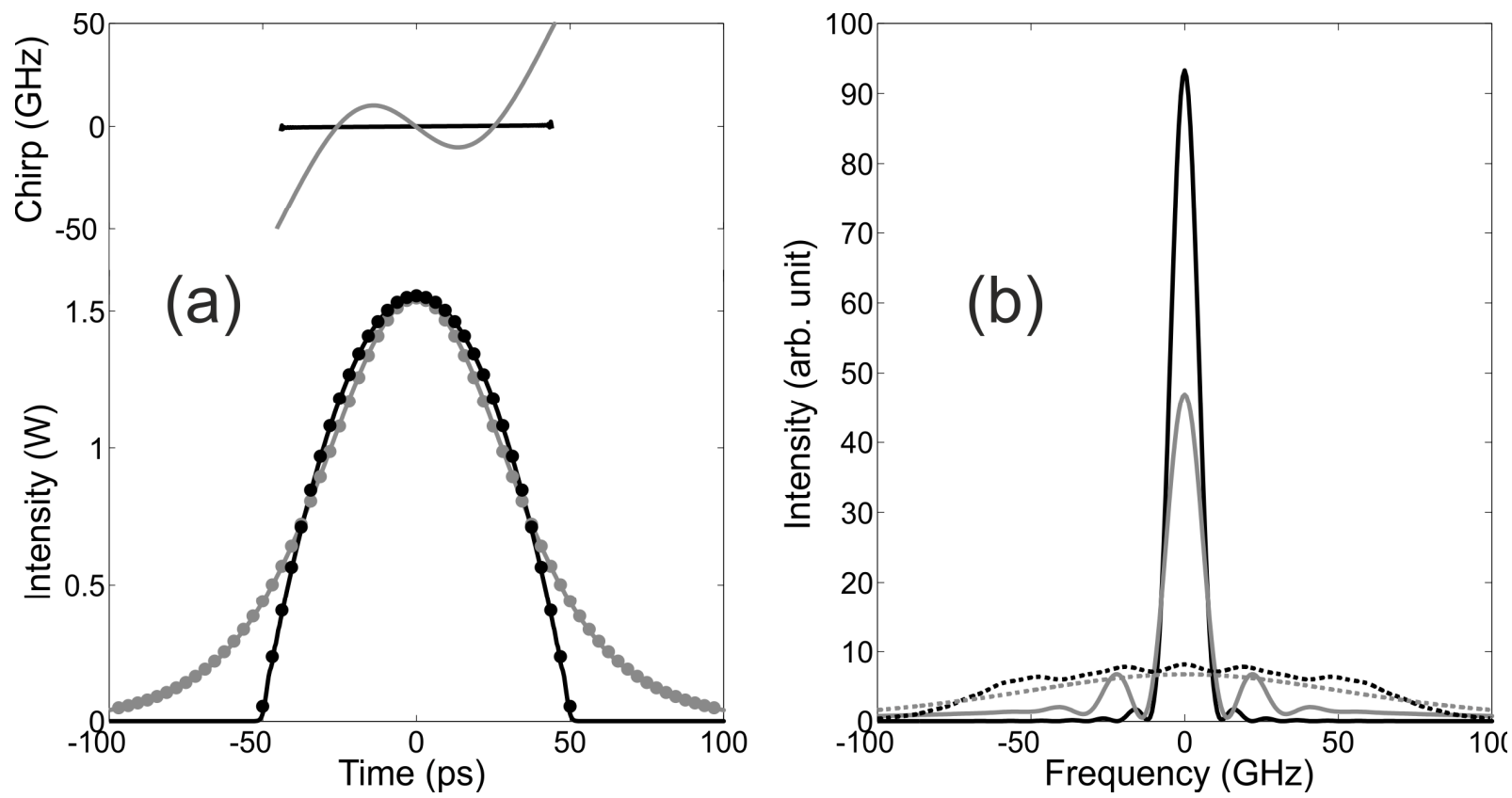

Fig. 3 : Simulation: (a) Temporal intensity and chirp profiles obtained for the optimum point of compression for hyperbolic secant and parabolic profiles (grey and black line respectively). The input temporal intensity profiles are plotted with full circles. (b) Spectral intensity profiles at the point of optimum compression for hyperbolic secant and parabolic profiles (grey and black solid lines respectively). The results are compared to the initial spectral plotted in dashed lines.

\section{Experiments}

\section{Experimental setup}

The experimental setup is sketched in Fig. 4. An initial picosecond pulse train is delivered by a modelocked erbium doped fiber laser running at a repetition rate of $10 \mathrm{GHz}$ at a central 
wavelength of $1552.5 \mathrm{~nm}$. The initial pulses are transform-limited with an hyperbolic secant intensity profile typical of soliton pulses and with a FWHM temporal duration of 2.4 ps. The repetition rate is then decreased down to $1.25 \mathrm{GHz}$ by means of an intensity modulator driven by a 1:7 duty cycle pulse sequence provided by a 10-Gbit/s electrical pattern generator. Decreasing the repetition-rate of the pulse train enables us to have access, for the same average power, to a higher peak-power. Note that this pulse picking technique has also the additional advantage to avoid any spectrum comb structure of $10-\mathrm{GHz}$ spaced lines. Indeed, with a $1.25 \mathrm{GHz}$ repetition rate and given our optical spectrum analyzer resolution, the resulting spectrum appears as continuous. A phase modulator driven by a sinusoidal beating at a frequency around $100 \mathrm{MHz}$ is also used to avoid the deleterious effects of Brillouin backscattering. The amplitude shaping is achieved by a liquid crystal on silicon (LCoS) device [5] that enables to tailor both the amplitude and phase spectral profiles of the incoming pulse train. Note however that in our case, we will only benefit from the amplitude shaping capabilities of the devices. Indeed, the linear spectral chirp that is targeted is too high to be directly synthesized by the LCoS device. Consequently, in order to imprint the linear chirp, we use a piece of anomalous standard single mode fiber with a length $\mathrm{L}_{\mathrm{SMF}}$ of $3900 \mathrm{~m}$ and a second-order dispersion $\beta_{2 \mathrm{SMF}}=-21.9 \mathrm{ps}^{2} / \mathrm{km}$. The fiber rigorously leads to a parabolic spectral phase $\beta_{2 \mathrm{SMF}} \mathrm{L}_{\mathrm{SMF}} \omega^{2} / 2, \omega$ being the radial frequency. Due to the high level of parabolic spectral phase, this leads in the temporal domain to the expected nearly quadratic temporal phase. The initial 2.4-ps pulses are thus stretched to a temporal duration of 70 ps. The resulting pulses are characterized in intensity by a fast oscilloscope with a $63 \mathrm{GHz}$ bandwidth, high enough to obtain the shape of the intensity profile. The low incoming power in the SMF and its low nonlinear coefficient ensure that no unwanted nonlinear effects affect the pulse propagation in this segment. Then the pulses are amplified to the required average power 
(up to $30 \mathrm{dBm}$ ) by a high-power erbium doped fiber amplifier (EDFA) and the output power is varied by an optical variable attenuator in order to avoid power-dependent nonlinear effects in the amplifier. The optical attenuator is controlled by a computer (PC) and synchronized to the optical spectrum analyzer, which enables us to obtain systematic mapping of the output spectral shape. Finally, the spectral narrowing takes place in a highly nonlinear fiber (commercially available HNLF distributed by OFS) with a length of $1 \mathrm{~km}$, an attenuation of $1 \mathrm{~dB} / \mathrm{km}$ and a nonlinear coefficient of $10 / \mathrm{W} / \mathrm{km}$. The chromatic dispersion is chosen to be normal $\left(\beta_{2}=9.610^{-4}\right.$ $\mathrm{ps}^{2} / \mathrm{m}$ is used) in order to prevent from any modulation instability process [11]. An optical spectrum analyzer (resolution of $5 \mathrm{GHz}$ ) has been used to record the resulting spectra.

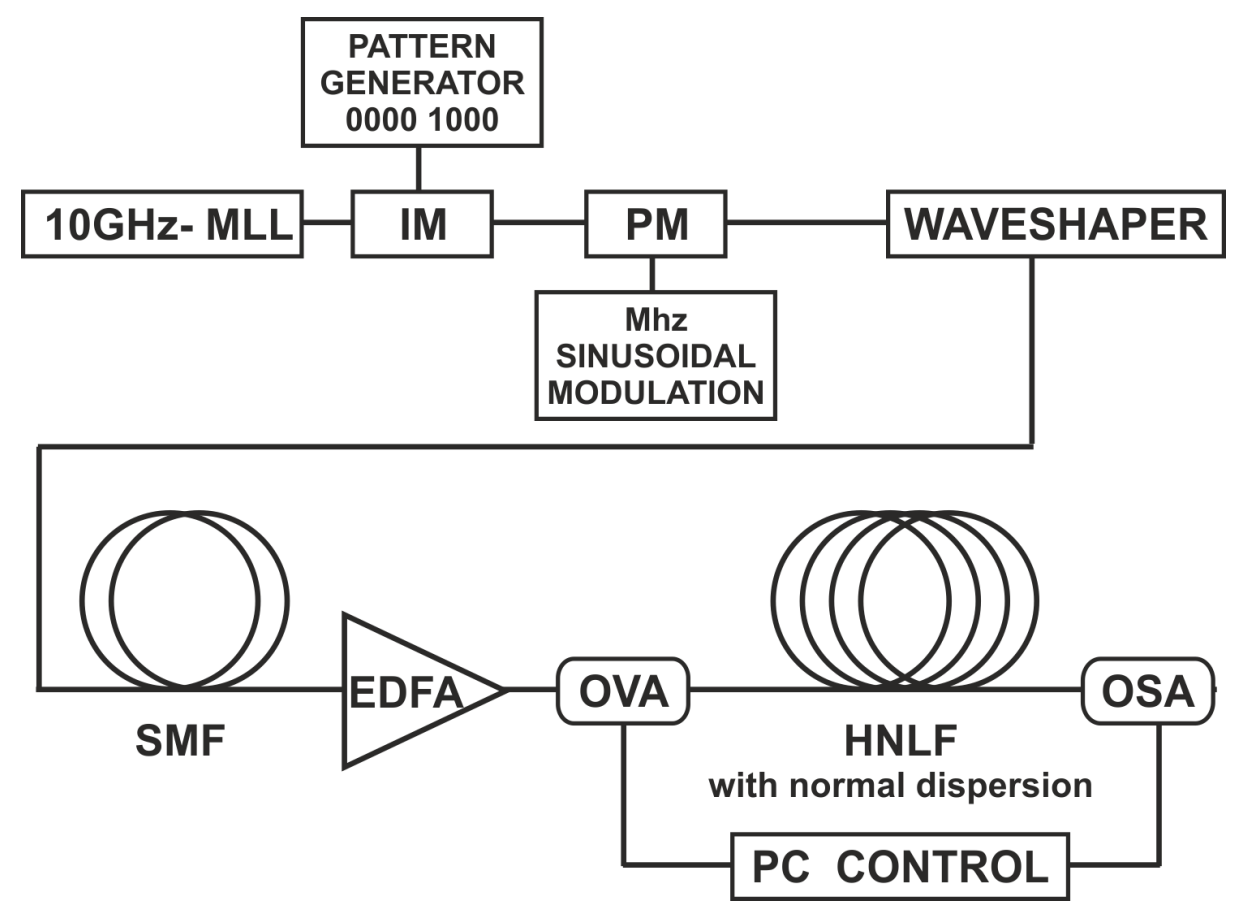

Fig. 4 : Experimental setup. MLL : modelocked laser ; IM : intensity modulator ; PM : phase modulator ; SMF : single mode fiber; EDFA : erbium-doped fiber amplifier; OVA : optical variable attenuator; HNLF : highly nonlinear fiber ; OSA : optical spectrum analyzer. 


\section{Experimental results}

The experimental temporal intensity profile recorded after pulse shaping and stretching is plotted in Fig. 5. We can clearly see that the initial intensity spectral shaping enables to efficiently shape the hyperbolic secant pulses into a parabolic profile. The impact of the power launched into the fiber is demonstrated in Fig. 6 where we have plotted similarly to Fig. 2, the output spectrum according to the input peak power of the incoming pulses. This map is made of 200 spectra. Those experimental results agree well with the qualitative trends that have been described in the previous section: the spectral narrowing is progressive and becomes optimum for input peakpowers close to $1.5 \mathrm{~W}$. The significant difference in behavior previously discussed between the two types of pulse shape is also confirmed.

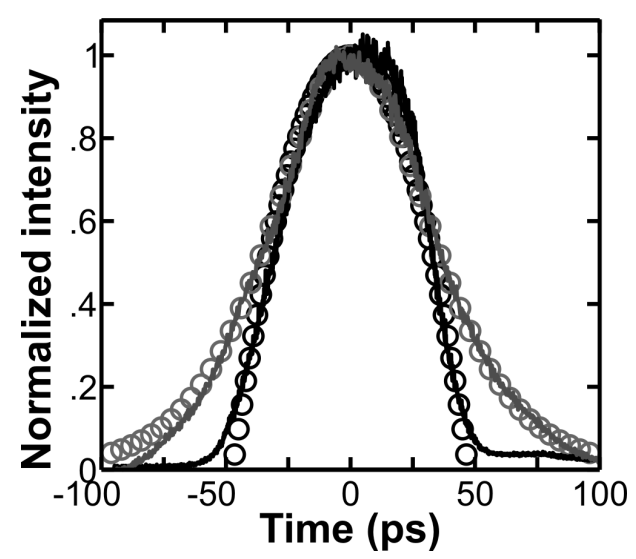

Fig. 5 : Initial pulse profiles after stretching in the 3.9-km long SMF segment. In absence of amplitude spectral shaping, the recorded intensity profile (grey line) is compared to a $\operatorname{sech}^{2}$ intensity fit (grey circles). Using an initial spectral shaping, the experimental results (black line) are compared to a parabolic intensity profile (black circles). 

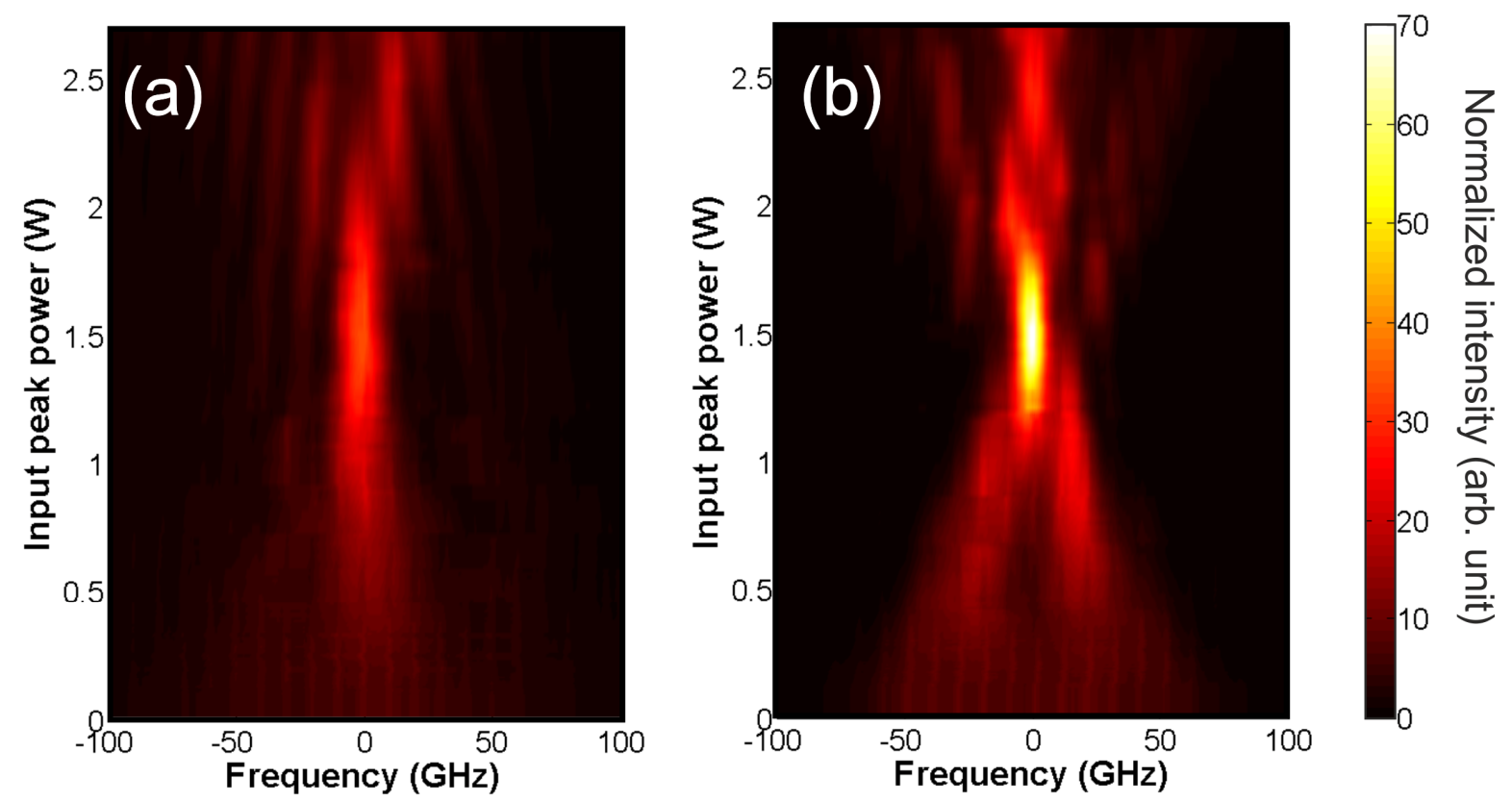

Fig. 6 : Experimental results: Evolution of the spectral profile as a function of the input peak power for an initial $\mathrm{sech}^{2}$ intensity profile (a) and for a parabolic intensity profile (b). Spectra are normalized so that the energy of the spectra equals 1 .

More details on the spectral shape of the recompressed pulses are given in Fig. 7a and stress that the parabolic shaped pulses lead to an enhanced spectral compression providing lower sidelobes and increased peak spectral power. Compared to input pulses, the power density has then been increased by a factor 9. Moreover, in agreement with numerical predictions, an improvement of spectral brightness by a factor 2.1 is achieved compared to the $\operatorname{sech}^{2}$ pulses. A compression factor ratio of 12.2 is also measured, consistent with the factor expected from the numerical simulations. Note however that this value is slightly impacted by the resolution of our OSA (we believe the real value of the compressed spectral width to be slightly lower). We have checked that the temporal intensity profiles of the output pulses were not affected by the compression process. Therefore, the resulting time-bandwidth product is found to be 0.745 , to be compared to the value of transform-limited parabolic pulse of 0.66 . We are thus only $12 \%$ higher 
than the bandwidth limited case. On the other side, for hyperbolic secant pulses, the resulting time-bandwidth product is more than twofold above the transform limit condition $(0.315$ for a transform-limited hyperbolic secant pulse).

Regarding the evolution of the pulse quality, the Strehl ratio demonstrates once again the superiority of the parabolic-shaped pulses with a Strehl ratio 3 times higher than in the sech pulse case. Finally, the evolution of the rms spectral width measured for the parabolic pulse shape as a function of input peak power confirms the parabolic law described in the previous section.
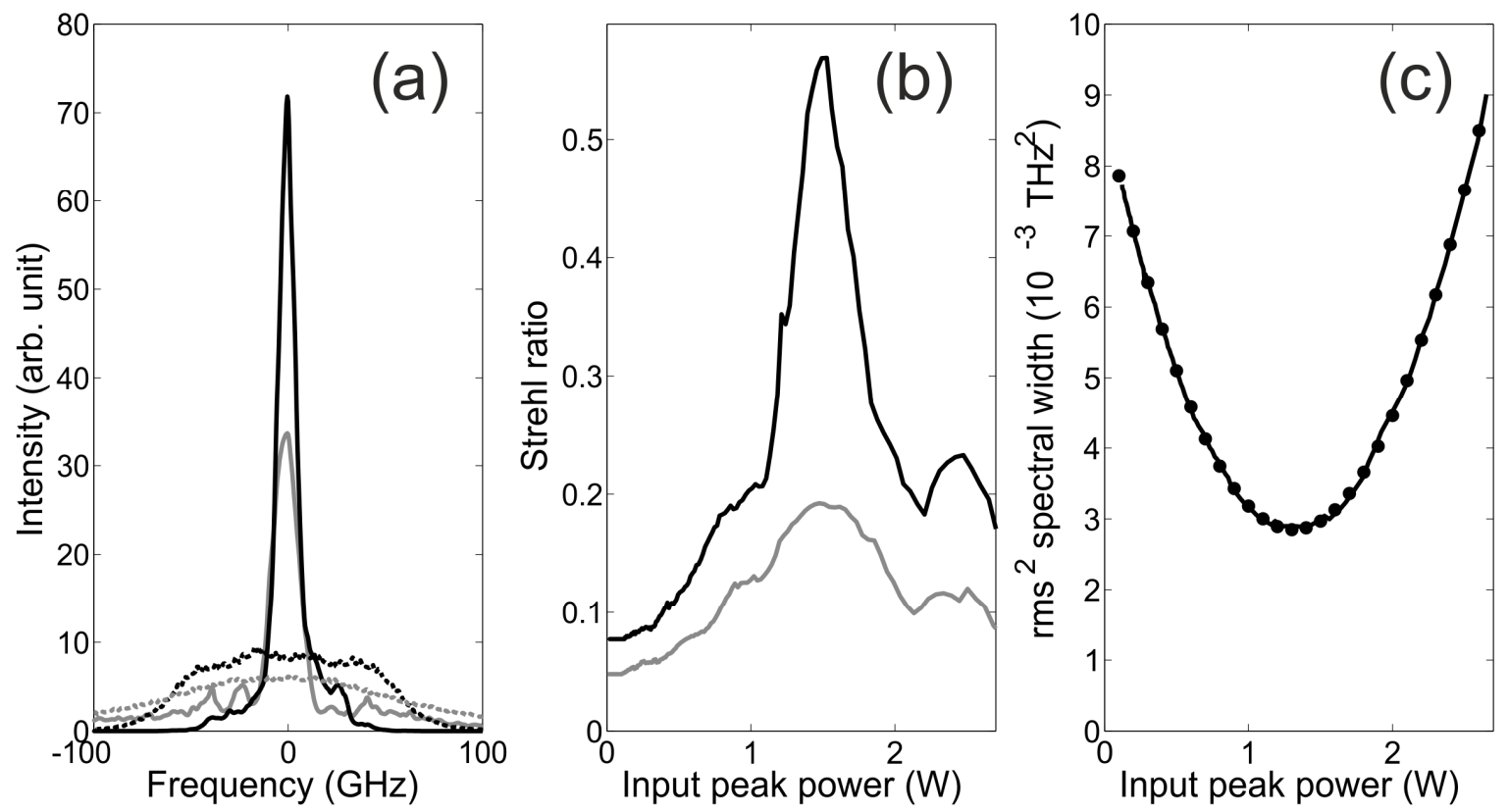

Fig. 7 : (a) Optical Spectra obtained at the optimum compression point (solid line) for a sech pulse (grey line) and parabolic shaped pulse (black line) and compared to the input spectra (dashed line). (b) Evolution of the Strehl ratio according to the initial peak-power for the parabolic pulse (solid black line) and sech pulse (solid grey line) (c) Evolution of the rms spectral width according to the input peak power for a parabolic pulse : experimental results (solid black line) are compared to a parabolic law.

\section{Discussion}

In our experiment, we have demonstrated a x12 compression ratio. To the best of our knowledge, this result represents a record for generation of near transform limited pulse: the previous works 
dealing with the same technique in a passive fiber have reported a compression factor below 10 $[9,10,12,13,16,17]$, except in $[11]$ but the high level of compression ratio was achieved at the expense of high sidelobes and pulses were far from being close to transform-limit. Regarding many aspects, the superiority of the shaped pulse has been definitively confirmed. However, the results have been below the theoretical predictions obtained for a parabolic pulse shape. In particular, higher substructures have been recorded, thus explaining the lower Strehl ratio equal to 0.57 instead of 1 . We attribute this mainly to the imperfect parabolic pulse shape that has been initially synthesized. Indeed the process is intrinsically sensitive to the gradient of the intensity profile and as can be seen in Fig. 5, some deviations from a perfect inverted parabola are observed. This temporal defect could have been suppressed using a feedback loop in the shaping process to iteratively optimize the input shape. This point is however beyond the scope of the present paper, our target being to confirm the benefits of parabolic pulses.

Higher compression factors could have been obtained using a longer stretching fiber. Given, the high ratio of $\beta_{2 \mathrm{SMF}} / \beta_{3 \mathrm{SMF}}$ in SMF fiber, the influence of third-order dispersion $\beta_{3 \mathrm{SMF}}$ can be neglected as a first approximation (the typical dispersion length linked to $\beta_{3 \mathrm{SMF}}$ is more than one order of magnitude higher than the fiber length under use) : for the picosecond pulse under investigation, the cubic phase induced by $\beta_{3 \mathrm{SMF}}$ is not as deleterious as what has been outlined when dealing with femtosecond pulses [10, 21]. If really needed, they can be compensated for using the phase tailoring ability of the LCoS device. Second- and third-order dispersion of the dispersion-flattened HNLF have also not been found to be here a limiting factor during the spectral compression process since dispersive effects have low influence on the narrow spectrum. 
However, some practical issues have to be taken into account. First, the strong temporal broadening of the input pulses should not lead to pulse overlapping in the wings of the pulses. Therefore, around half of the bit time seems to be an upper limit to the stretching that should be achieved. Then, increasing the temporal pulse duration according to (7) requires a higher peak power and therefore a higher average power. A first limitation is of course the power capacity of the EDFA. But another stringent effect is the Brillouin backscattering [22]. Indeed, if Brillouin backscattering is often fully negligible for optical spectra of several tens of $\mathrm{GHz}$, in our configuration, due to the compression process that strongly increases the power spectral density, Brillouin backscattering becomes a stringent issue that we have had to overcome by means of an additional phase modulation.

\section{Conclusion}

We have demonstrated the spectral compression of picosecond pulses in an all-fiber configuration at telecommunication wavelengths. Using widely available standard single mode fiber as a stretcher to impose the initial linear chirp, a spectral narrowing by a factor above 12 has been achieved for output pulses close to the Fourier transform limit. This compression has been based on a kilometer long highly nonlinear fiber with W-level peak powers and has involved pulse energies well below the $\mathrm{nJ}$ level and is compatible with $\mathrm{GHz}$ operation. An initial parabolic pulse shaping has also been shown to significantly improve the results. The quality of the compressed spectra has indeed been enhanced with much lower substructures and an improved Strehl ratio. 


\section{Acknowledgements}

We acknowledge the support of the Agence Nationale de la Recherche (ANR SOFICARS, grant ANR-07-RIB-013-03) and Conseil Regional de Bourgogne (PARI Photcom). We also thank Philippe Morin, Kamal Hammani, Guy Millot, Charles-Henri Hage and Benoit Frisquet for their help and for fruitful discussions.

\section{References}

1. R. H. Stolen, and Q. Lin, "Self-phase modulation in silica optical fibers," Phys. Rev. A 17, 1448-1453 (1978).

2. Z. Yousoff, P. Petropoulos, F. Furusawa, T. M. Monro, and D. J. Richardson, "A 36channel $\mathrm{x} 10-\mathrm{GHz}$ spectrally sliced pulse source based on supercontinuum generation in normally dispersive highly nonlinear holey fiber," IEEE Photon. Technol. Lett. 15, 1689-1691 (2003).

3. W. J. Tomlinson, R. H. Stolen, and C. V. Shank, "Compression of optical pulses chirped by self-phase modulation in fibers," J. Opt. Soc. Amer. B 1, 139-149 (1984).

4. F. Parmigiani, C. Finot, K. Mukasa, M. Ibsen, M. A. F. Roelens, P. Petropoulos, and D. J. Richardson, "Ultra-flat SPM-broadened spectra in a highly nonlinear fiber using parabolic pulses formed in a fiber Bragg grating," Opt. Express 14, 7617-7622 (2006).

5. A. M. Clarke, D. G. Williams, M. A. F. Roelens, and B. J. Eggleton, "Reconfigurable Optical Pulse Generator Employing a Fourier-Domain Programmable Optical Processor," J. Lightw. Technol. 28, 97-103 (2010).

6. D. N. Schimpf, J. Limpert, and A. Tünnermann, "Controllong the influence of SPM in fiber-based chirped-pulse amplification systems by using an actively shaped parabolic spectrum," Opt. Express 15, 16945-16953 (2007).

7. C. Finot, J. M. Dudley, B. Kibler, D. J. Richardson, and G. Millot, "Optical parabolic pulse generation and applications," IEEE J. Quantum Electron. 45, 1482-1489 (2009).

8. N. L. Markaryan, L. K. Muradyan, and T. A. Papazyan, "Spectral compression of ultrashort laser pulses," Kvantovaya Elektron. 18, 865-867 (1991).

9. S. A. Planas, N. L. Pires Mansur, C. H. Brito Cruz, and H. L. Fragnito, "Spectral narrowing in the propagation of chirped pulses in single-mode fibers," Opt. Lett. 18, 699-701 (1993).

10. M. Oberthaler, and R. A. Höpfel, "Spectral narrowing of ultrashort laser pulses by selfphase modulation in optical fibers," Appl. Phys. Lett. 63, 1017-1019 (1993).

11. E. R. Andresen, J. Thogersen, and S. R. Keiding, "Spectral compression of femtosecond pulses in photonic crystal fibers," Opt. Lett. 30, 2025-2027 (2005).

12. J. P. Limpert, T. Gabler, A. Liem, H. Zellmer, and A. Tünnermann, "SPM-induced spectral compression of picosecond pulses in a single-mode Yb-doped fiber amplifier," Appl. Phys. B 74, 191-195 (2002). 
13. E. R. Andresen, V. Birkedal, J. Thogersen, and S. R. Keiding, "Tunable light source for coherent anti-Stockes Raman scattering microspectroscopy based on the soliton self-frequency shift," Opt. Lett. 31, 1328-1330 (2006).

14. C. Finot, A. Guenot, and P. Dupriez, "Spectral compression of optical parabolic similaritons," Ann. Phys. Fr 32, 71-74 (2007).

15. C. Finot, F. Parmigiani, P. Petropoulos, and D. J. Richardson, "Parabolic pulse evolution in normally dispersive fiber amplifiers preceding the similariton formation regime," Opt. Express 14, 3161-3170 (2006).

16. E. R. Andresen, J. M. Dudley, C. Finot, D. Oron, and H. Rigneault, "Transform-limited spectral compression by self-phase modulation of amplitude shaped pulses with negative chirp," Opt. Lett. 36, 707-709 (2011).

17. M. Rusu, and O. G. Okhotnikov, "All-fiber picosecond laser source based on nonlinear spectral compression," Appl. Phys. Lett. 89, 091118 (2006).

18. T. Hirooka, M. Nakazawa, and K. Okamoto, "Bright and dark $40 \mathrm{GHz}$ parabolic pulse generation using a picosecond optical pulse train and an arrayed waveguide grating," Opt. Lett. 33 (2008).

19. Y. Zaouter, D. N. Papadopoulos, M. Hanna, F. Druon, E. Cormier, and P. Georges, "Third-order spectral phase compensation in parabolic pulse compression," Opt. Express 15, 9372-9377 (2007).

20. D. Anderson, and M. Lisak, "Analytic study of pulse broadening in dispersive optical fibers," Phys. Rev. A 35, 184-187 (1987).

21. A. A. Kutuzyan, T. G. Mansuryan, G. L. Esayan, R. S. Akopyan, and A. Muradyan, "Dispersive regime of spectral compression," Quantum Electronics 38, 383 (2008).

22. R. G. Smith, "Optical power handling capacity of low loss optical fibers as determined by stimulated Raman and Brillouin scattering," Appl. Opt. 11, 2489-2494 (1972). 